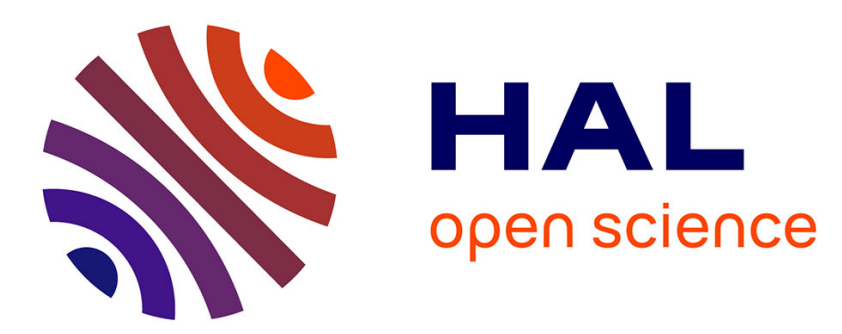

\title{
Fusion of Perception and V2P Communication Systems for Safety of Vulnerable Road Users
}

\author{
Pierre Merdrignac, Oyunchimeg Shagdar, Fawzi Nashashibi
}

\section{To cite this version:}

Pierre Merdrignac, Oyunchimeg Shagdar, Fawzi Nashashibi. Fusion of Perception and V2P Communication Systems for Safety of Vulnerable Road Users. IEEE Transactions on Intelligent Transportation Systems, 2016. hal-01399150

\section{HAL Id: hal-01399150 \\ https://inria.hal.science/hal-01399150}

Submitted on 18 Nov 2016

HAL is a multi-disciplinary open access archive for the deposit and dissemination of scientific research documents, whether they are published or not. The documents may come from teaching and research institutions in France or abroad, or from public or private research centers.
L'archive ouverte pluridisciplinaire HAL, est destinée au dépôt et à la diffusion de documents scientifiques de niveau recherche, publiés ou non, émanant des établissements d'enseignement et de recherche français ou étrangers, des laboratoires publics ou privés. 


\title{
Fusion of Perception and V2P Communication Systems for Safety of Vulnerable Road Users
}

\author{
Pierre Merdrignac $^{1,2}$, Oyunchimeg Shagdar ${ }^{1,2}$, and Fawzi Nashashibi ${ }^{1}$
}

\begin{abstract}
With cooperative intelligent transportation systems (C-ITS), vulnerable road users (VRU) safety can be enhanced by multiple means. On one hand, perception systems are based on embedded sensors to protect VRUs. However, such systems may fail due to the sensors' visibility conditions and imprecision. On the other hand, Vehicle-to-Pedestrian (V2P) communication can contribute to the VRU safety by allowing vehicles and pedestrians to exchange information. This solution is, however, largely affected by the reliability of the exchanged information, which most generally is the GPS data. Since perception and communication have complementary features, we can expect that a fusion between these two approaches can be a solution to the VRU safety. In this work, we propose a cooperative system that combines the outputs of communication and perception. After introducing theoretical models of both individual approaches, we develop a probabilistic association between perception and V2P communication information by means of multi-hypothesis tracking (MHT). Experimental studies are conducted to demonstrate the applicability of this approach in real-world environments. Our results show that the cooperative VRU protection system can benefit of the redundancy coming from the perception and communication technologies both in line-of-sight (LOS) and nonLOS (NLOS) conditions. We establish that the performances of this system are influenced by the classification performances of the perception system and by the accuracy of the GPS positioning transmitted by the communication system.
\end{abstract}

Index Terms-Vulnerable Road Users, Cooperative ITS, Vehicle-to-Pedestrian (V2P), Information Fusion.

\section{INTRODUCTION}

$\mathbf{P}$ ROTECTING vulnerable road users (VRUs) has been a topic of interest for the past years. VRUs are defined as "non-motorized road users, such as pedestrians and cyclists and persons with disabilities or reduced mobility and orientation" [1]. According to the report on road safety provided by the World Health Organization [2], half of the fatalities due to traffic accidents concern VRUs. Cooperative Intelligent Transportation Systems (C-ITS) are proposing solutions to enhance road safety by sensors data processing, i.e. perception, and by enabling information exchange, i.e. communication, between vehicles $(\mathrm{V} 2 \mathrm{~V})$, vehicles and infrastructure (V2I), and, vehicles and pedestrians (V2P).

A cooperative system can benefit from the knowledge of every individual user to detect potential collision on vehicles and/or VRUs side, especially in urban environments as illustrated in Fig. 1.

1 P. Merdrignac, O. Shagdar, and, F. Nashashibi are with RITS ProjectTeam, INRIA Paris, 2 rue Simone Iff, CS 42112, 75589, Paris Cedex 12, FRANCE. name. surnamedinria.fr

${ }^{2}$ P. Merdrignac, and, O. Shagdar are with Institut VEDECOM, 77, rue des Chantiers, 78000, Versailles, FRANCE.

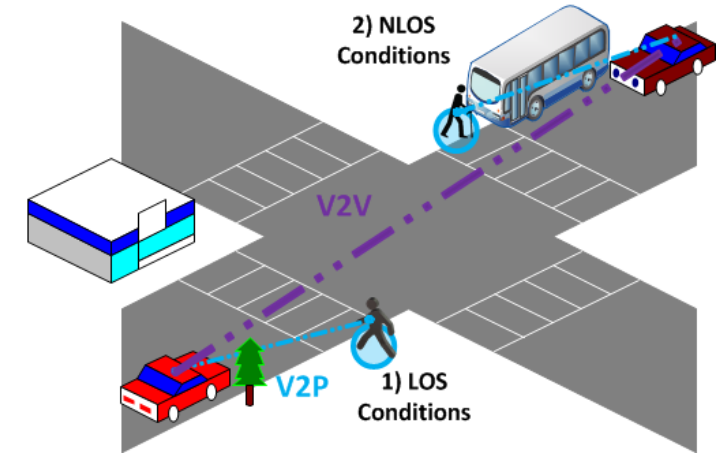

Fig. 1: Cooperative safety in urban environment.

Relying on embedded sensors data has been a first solution at very close range and low speed [3] to ensure the safety of VRUs who are visible from the vehicles, i.e., in Lineof-Sight (LOS) w.r.t. the vehicle, as shown in situation 1) of Fig. 1. However, more recent studies have demonstrated the need for new methods in order to tackle more complex cases such as pedestrians not visible from the vehicles [4] [5], i.e., in Non LOS (NLOS) conditions as shown in situation 2) of Fig. 1. Vehicular communication is able to disseminate information beyond the sensor's field of view (FOV), hence, it has a potential to improve road safety in both LOS and NLOS conditions.

In this paper, we introduce a new cooperative system based on both perception and V2P communication for protection of VRUs. On vehicles' side, perception relies on laser data to detect, classify and locate road obstacles situated in the sensors' FOV [6], [7]. On VRUs' side, user's location is estimated from GPS data and is transmitted to surrounding vehicles [8]. Hence, fusing information from perception, i.e. obstacles class and location, with the information from V2P communication, i.e. GPS data and VRUs' identifiers, is the main challenge. The proposed system has to distinguish the following cases:

- a perceived obstacle is also a communicating obstacle,

- a communicating obstacle is not detected by the perception system,

- a perceived obstacle is not communicating.

Here, fusion is tackled by a new probabilistic method inspired from multi-target tracking and we demonstrate how this approach handles both situations of Fig. 1.

The rest of the paper is organized as follows. Section II highlights the related work. Section III introduces perception and V2P communication systems for VRU protection and 
section IV details our probabilistic information fusion method. Experimental evaluation of our proposal is conducted in Section V. Finally, Section VI concludes our paper.

\section{RELATED WORK}

\section{A. Perception based VRU protection}

Detection of VRUs has been an active research topic since the early 2000s. In 2007, Gandhi et al. [3] proposed a large review of perception based systems for protection of VRUs. On one hand, active sensors such as radars [9]-[11], ultrasonic sensors [12], laser rangefinders [6], [7], [13]-[15] have been shown to be efficient to detect and locate obstacles situated in their FOV, but lack of precision to classify the detected obstacles. On the other hand, video cameras are natural sensors to recognize VRUs surrounding a vehicle both in illuminated scenes [4], [16]-[18] and night conditions [19], [20], but are weak to estimate precisely the relative position of a detected obstacle. In 2010, Gerònimo et al. [4] highlight that the number of false alarms is still too high in the current sensor based pedestrian detection systems to allow their deployment in commercial vehicles and propose to explore multi-class classification, 3D sensing, multi-model training as well as pedestrian tracking to improve their reliability.

In [5], Dollar et al. conducted an evaluation of 16 existing vision based pedestrian classifiers and showed that the performances of all the classifiers degrade sharply when the visibility condition (up to small scale and full occlusion) becomes worse. In the cases of heavy occlusion, nearly all pedestrians are missed.

In summary, despite large efforts, perception based VRU protection systems are not able to work perfectly in all the situations encountered in cities, especially when a VRU is strongly occluded by another obstacle. In such condition, only the occluding object can be detected using perception and other approaches such as communication have to be considered.

\section{B. Communication based VRU protection}

Communication systems have the potential to enhance road safety by allowing road users to exchange content rich data [21]-[23] and by activating environment based messages [24].

Standards are required to ensure a compatibility between communication entities, called ITS stations, from different manufacturers. Up to now, standardisation activities have put a strong focus on V2V and V2I communications, especially in Europe [25]-[27], and specified IEEE 802.11p [28] for vehicular communication. V2P has recently appeared as a specific communication mode due to the strong requirement on communication delay and energy consumption at the VRUs side [21], [23], [29], and, it is not yet standardized.

Limited battery life of VRU hand-held devices has to be considered as a constraint in the design of V2P communication protocols [8], [30], [31]. The common objective of these approaches is to minimize messages emitted from the VRUs by assessing the collision risk and by relying either on centralized [31] or distributed [8], [30] communication technologies.
In our previous works [8], [23], we introduced a pedestrian protection system based on distributed communication. Here, pedestrians receiving a cooperative awareness message (CAM) [22] from a vehicle evaluate whether they are in the near future trajectory of this vehicle or in a geographical area that is hidden w.r.t. the vehicle to trigger V2P communication. Vehicles receiving messages from pedestrians store the information content in their local dynamic map (LDM) [32].

To summarize, despite some efforts for establising V2P communication, further work is needed to avoid packets loss due to a high number of communication nodes (vehicles and VRUs) and/or due to signal blockage in NLOS conditions [8], [23]. Relying on geographical routing algorithms such as GeoNetworking [33] could be a future approach to disseminate V2P messages. In this paper, the data exchanged for VRU protection by V2P communication are detailed.

\section{Information fusion in C-ITS}

With C-ITS, multi-source information is fused in order to overcome the limitations of standalone systems. For example, extending perception using cooperative methods has been the main interest in many recent works [34]-[39].

Global Positioning System (GPS) [40] is installed in many of current devices (vehicles, smartphones,...) to provide absolute coordinates which can be converted to a local reference frame using Lambert conformal conic projection [39]. However, many perturbations on the GPS signals (imprecise clock synchronisation, perturbation from the atmosphere, urban canyons,...) lead to errors of 5 to $10 \mathrm{~m}$ [41] which are too large to fulfill the requirements of safety applications.

Cooperative sensors based on IEEE 802.15.4 technology have been developed during the projects WATCH-OVER [42] and Ko-TAG [43]. These sensors measure precisely the relative positioning between vehicles and VRUs by calculating the time of flight of the emitted signal. Despite low power consumption, these devices require perfect clock synchronization. Fusion of such cooperative localization with embedded sensors data was also investigated during these projects [44], [45], but the proposed approaches are still inefficient in NLOS conditions due to the reduced precision of localization and the inability of detecting hidden VRUs in sensors data.

Information fusion requires the management of data obtained from ego vehicle perception and from vehicular communication. In multi-target tracking, solutions such as joint probabilistic data association (JPDA) [46] and multi-hypothesis tracking (MHT) [47] can manage tracks of obstacles observed by multiple sensors. In C-ITS, such approaches have been applied either in probabilistic [6] or evidential [37]-[39] framework. Contrary to the work of Demmel [39] where homogeneous sources are fused by means of MHT, our system has to consider fusion between heterogeneous sources since the embedded VRU perception and V2P communication provide totally different information.

In summary, current approaches for cooperative VRU protection systems are still not able to handle LOS as well as NLOS conditions. Thus, we introduce a new approach for VRU protection which relies on the capacity of perception to 
detect, localize and classify obstacles located in the sensors' FOV and the capacity of V2P communication to disseminate information beyond the sensors' FOV, i.e., to long range and occluded zones. Our approach takes into account the advantages of perception to estimate dynamic parameters such as speed and velocity and the advantages of V2P communication to transmit static parameters such as the user class.

\section{PERCEPTION AND COMMUNiCATION FOR VRU PROTECTION}

Improving VRU safety in urban environments requires a protection system that works in different situations as illustrated in Fig. 1. In such cases, perception is used to detect, localize and classify VRUs who are visible w.r.t the vehicle and V2P communication brings data exchange (user position and velocity, user class,...) and extends the FOV defined by embedded sensors. Hence, we propose a cooperative approach that can jointly alert vehicles and VRUs of potential collisions. Fig. 2 illustrates the proposed system where vehicles are equipped with perception (1), V2P communication (2), fusion of perception and V2P communication data (4) and collision risk evaluation (5), and VRUs are equipped with V2P communication (3) and collision risk evaluation (5).

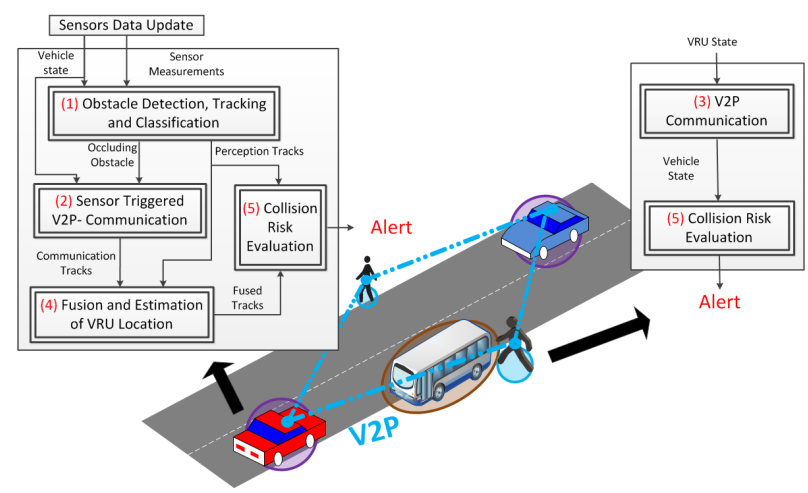

Fig. 2: Overall cooperative protection system description

In this section, we focus on perception and V2P communication separately and evaluate their limitations before introducing the fusion algorithm.

\section{A. Perception system for VRU protection}

1) Assumption: Vehicles are equipped with laser range finders for perception. Such sensors describe the environment in $\mathrm{x}-\mathrm{y}$ coordinates by means of impact points.

2) Data processing: Obstacles position and velocity are estimated by means of point cloud clustering and Kalman filtering. Geometric features are used to classify the detected obstacles [6], [7].

3) Output: This system output tracks of VRUs having a unique identifier across time. Each track estimates the relative position, velocity and class of a detected obstacle.

\section{B. V2P Communication for VRU protection}

1) Assumption: Vehicles and VRUs are equipped with radio communication systems. As introduced in [23], V2P communication requires low transmission delay by means of distributed communication protocols such as ad hoc mode with Wi-Fi protocols (IEEE 802.11) or Device-to-Device (D2D) communications with LTE protocol. In this paper, Wi-Fi protocol IEEE $802.11 \mathrm{~g}$ was available on both vehicle side and VRU hand-held device and was used during the tests, but other distributed communication media could be used with the proposed system.

2) Data processing: As described in [8], our V2P communication protocol relies on CAM transmitted by vehicles for informing VRUs and for triggering communication on the VRUs side.

A vehicle has to send its position as its latitude (Lat.), longitude (Long.) and orientation $\left(\theta_{v e h}\right)$. The generation time $t_{v e h}$ of this data is also important. Finally, vehicle speed, $v_{v e h}$, and yaw rate, $\omega_{v e h}$, as well as the parameters of width, $W$, and length, $L$, are necessary to evaluate the collision risk [23]. Table I summarizes mandatory parameters that have to be emitted by the vehicle.

TABLE I: Parameters emitted by the vehicle

\begin{tabular}{|c|c|c|}
\hline Description & Notation & \\
\hline Identifier & ID & \\
Latitude & Lat. & \\
Longitude & Long. & $\Omega$ \\
Orientation & $\theta_{v e h}$ & $\Xi$ \\
Generation time & $t_{v e h}$ & $\S$ \\
Radial velocity & $v_{v e h}$ & $\Xi$ \\
Yaw rate & $\omega_{v e h}$ & $\Xi$ \\
Vehicle length & $W$ & \\
Vehicle width & $L$ & \\
\hline
\end{tabular}

Once a VRU receives a CAM, it evaluates if the pedestrian is in a geographical dissemination area for this message. If positive, a P2V message, which has to contain the parameters described in Table II, is emitted by the VRU.

TABLE II: Parameters emitted by the VRU

\begin{tabular}{|c|c|c|}
\hline Description & Notation & \\
\hline Identifier & ID & \\
\hline Latitude & Lat. & N \\
\hline Longitude & Long. & $<$ \\
\hline VRU orientation & $\theta_{v r u}$ & 3 \\
\hline Generation time & $t_{v r u}$ & مी \\
\hline Velocity & $v_{v r u}$ & कृ \\
\hline VRU Class & $C_{v r u}$ & \\
\hline
\end{tabular}

3) Output: The vehicle receiving such a P2V message transforms latitude and longitude in its own local coordinate system to estimate the relative position of the communicating pedestrian [8], [39]. In addition, every communicating obstacle has a unique identifier obtained from the installation of the safety application [23]. Finally, the vehicle LDM is updated with the received $\mathrm{V} 2 \mathrm{P}$ data and can provide a list of the VRUs which have communicated. Within the LDM, obstacles location is predicted when the VRU protection system accesses to the list of VRUs for collision risk evaluation.

\section{Qualitative evaluation of Perception and V2P communica- tion systems}

In [8], we studied the capacity of perception and communication systems for VRU protection. Let us consider the 
test scenario where a vehicle approaches a pedestrian on a straight line to evaluate qualitatively the results obtained by the perception system and by the V2P communication system.

Figure 3 displays two snapshots for two experiments of perception and V2P communication systems 1) when the pedestrian is visible from the vehicle (Fig. 3a) and 2) when the pedestrian is occluded (Fig. 3b).

Here, the black rectangle illustrates the ego vehicle that tries to detect the pedestrian. In addition, the environment is expressed in the local coordinates of the ego vehicle, the $\mathrm{x}$ axis is along the longitudinal direction and the $y$-axis is along the lateral direction. Laser data are plotted as black crosses and provide a view of the current environment.

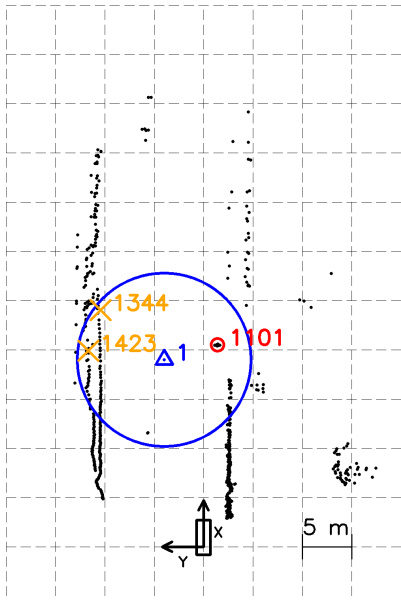

(a) LOS conditions.

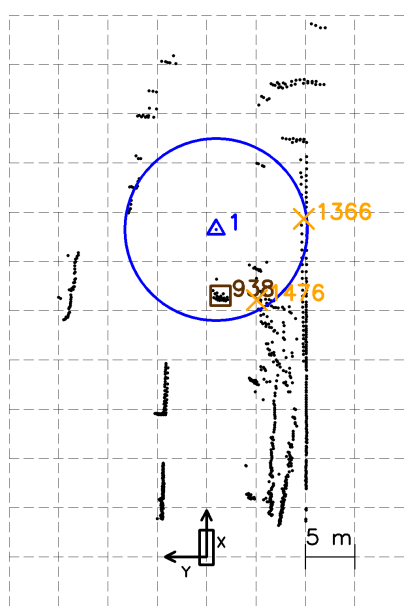

(b) NLOS conditions.
+ Laser impact $O$ Perception based VRU detection $\square$ Occluding obstacle $X$ Other obstacle with perception $\Delta$ Communication based VRU detection

Fig. 3: Obstacle detection in various conditions.

As can be seen in Fig. 3a, while the pedestrian is detected in the front right side of the vehicle (red circle), the communication system announces his position as in front left of the vehicle (blue triangle). Since positioning of the laser system is reliable, this is the case where, the communication system has an error due to the inaccuracy of the GPS data.

In Fig. 3b, the pedestrian is behind an obstacle (brown square). Here, the communication system could "detect" the pedestrian but the perception system could not. Nevertheless, the perception system could successfully detect the occluding obstacle.

To summarize, the limitations of the individual systems are:

- the reliability of the $\mathrm{V} 2 \mathrm{P}$ communication system is degraded due to the inaccuracy of the GPS data (Fig. 3a),

- the ability to detect pedestrians is limited by FOV of the sensors for perception systems,

- the ability to detect pedestrians is limited for the perception system if an obstacle blocks the line-of-sight to the pedestrian (Fig. 3b).

Consequently, information fusion between perception and $\mathrm{V} 2 \mathrm{P}$ communication is necessary to benefit from the advantages of both systems, i.e., the ability of V2P communication to inform of the presence of a VRU and the ability of perception to precisely localize obstacles.

\section{Fusion between Perception and V2P Communication}

The objective of the fusion stage is to determine whether a communicating VRU is visible or is hidden behind an obstacle. In the former case, the relative location of the communicating VRU can be improved by considering the "perceived" VRU as, in the latter case, the relative location of the communicating VRU cannot be precisely estimated.

In Fig. 3, a communicating pedestrian (blue triangle) send its GPS position by V2P communication. Due to the large imprecision of this sensor, the VRU can be located anywhere inside the uncertainty circle calculated from the GPS measurement. Such circle defines a gate where the different obstacles (red circle, brown square and orange cross) given by the perception module could have generate this V2P communication data.

Fig. 3a illustrates LOS conditions where the communicating pedestrian (blue triangle) is also detected by the perception system (red circle). In this case, the system may associate the communicating pedestrian either with the correct one (red circle) or with other obstacles (orange cross) that looks like pedestrians.

Fig. $3 \mathrm{~b}$ illustrates NLOS conditions where the communicating pedestrian stands behind a parked vehicle (brown square). In this case, the pedestrian is not seen by the perception system, and, the system should not associate him to any of the two obstacles situated within the gate.

Finally, the main goal is to establish which perception data has generated a communication data in LOS conditions and to establish that the communicating pedestrian is hidden in NLOS conditions. Consequently, the fusion stage has to be able to handle both LOS and NLOS cases to take the correct decision.

These cases can be seen as an association problem between the tracked data obtained from perception and V2P communication and can be tackled by means of multi-hypothesis tracker (MHT) [47]. Thus, we decided to apply MHT with $\mathrm{V} 2 \mathrm{P}$ communication and perception tracks where association hypotheses are generated and describe the following situations:

- the possible associations of communication tracks with perception tracks located inside the gate,

- the case of communication tracks not detected by the perception system.

Figure 4 describes the fusion stage between perception and V2P communication. In this algorithm, association hypotheses are generated by a loop on the V2P communication tracks. Indeed, for every V2P communication track, the list of association hypotheses is updated with the perception obstacles which are situated within the gate defined by the GPS data.

Then, a probability is calculated for every hypothesis based of the likelihood of such association between perception and V2P communication tracks.

After the fusion process, the state of VRUs is estimated by selecting the hypothesis with the highest probability. 


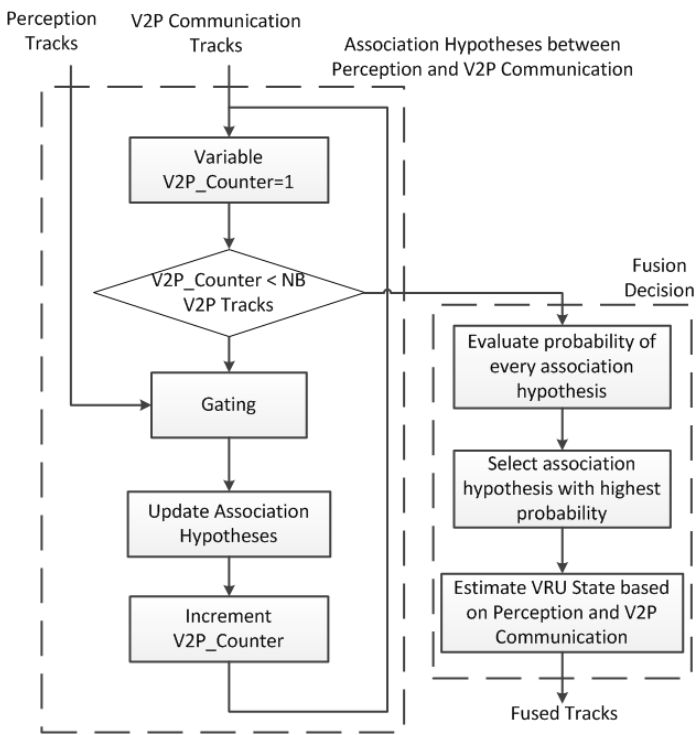

Fig. 4: Flow chart of fusion between perception and V2P communication

\section{MULTI HyPothesis TRACKER APPLIED FOR VRU PROTECTION}

In this section, we provide the theoretical aspects of the proposes fusion approach. First, we introduce a generic metric to evaluate VRU protection systems. Then, perception and V2P communication systems are modeled in terms of detection, classification and relative positioning. These three criteria are finally of importance for perception/V2P communication fusion established by means of a MHT association.

\section{A. Performance metric for VRU protection systems}

In order to evaluate the performance of our fusion algorithm, we defined in [8] a distance metric which has the capability to jointly evaluate detection and positioning errors. Such metric does not necessarily represent whether an accident is going to happen or not, but it can establish the performance of a VRU detection system that will be used for collision prevention.

Then, the fusion error $E_{f u s}$ is defined as the mean of the distance calculated over multiple trials for a given detection system. $E_{f u s}$ is expressed as two terms in eq. (1). The first term represents the mean positioning error and the second term is the probability of non detection weighted by a parameter $c$, a cut-off distance representing a maximum error value.

$$
E_{\text {fus }}=P_{d} \times M P E+c \times\left(1-P_{d}\right)
$$

where $M P E$ is the mean distance error and $P_{d}$ is the probability of detection.

This fusion error can be independently applied to evaluate the performances of a perception based protection system, a V2P communication based protection system and a cooperative protection system.

\section{B. Modeling Perception and V2P communication}

For simplicity, we introduce the three following criteria for modeling perception and V2P communication systems:
- detection, i.e., the capacity to detect a given obstacle,

- classification, i.e., the capacity to assign the correct class (pedestrian, vehicle,...) to a detected obstacle,

- relative positioning, i.e., the capacity to estimate the relative position of a given obstacle w.r.t. the ego vehicle.

1) Detection probability: A perception system can detect an obstacle (pedestrian in our case), if the obstacle is in the sensor's FOV. For laser-based perception systems, the detection is possible if the distance to the obstacle, $d$, is less than the sensor range, $d_{m}$ :

$$
P_{d}^{\text {per }}(d)= \begin{cases}1, & \text { if } d \leq d_{m} \\ 0, & \text { otherwise }\end{cases}
$$

In [8], we presented a more complex model that considers the expected obstacle width, but it was shown this model is approximately the sensor FOV for small obstacles such as VRUs.

For the communication system, we can consider that a VRU is "detected" at his current state if the vehicle receives a packet from this VRU. Therefore, the VRU detection capability of the communication system is expressed by the successful packet reception probability. Assuming that the bit error rate (BER) for individual bits of a packet are equal, the pedestrian detection probability for a given data packet is:

$$
P_{d}^{c o m}=(1-B E R)^{L_{p}}
$$

Here, $L_{p}$ is the packet length and BER is a function of the SNR (signal to noise ratio).

2) Classification: Classification with the perception system is done by comparing models of road obstacles to features extracted from the sensors data [3]. Using statistical machine learning, a probability $p_{j}$ of being of a class $C_{j}$ is assigned to a detected obstacle [7] and classification with a perception system is modelled as follows

$$
P_{c}^{p e r}\left(C_{j}\right)=p_{j}
$$

With V2P communication, a VRU can explicitly indicate the user class $C_{v r u}$ in the exchanged message. Assuming no one is transmitting false information, the probability of assigning a class $C_{j}$ to the VRU is as follows

$$
P_{c}^{\text {com }}\left(C_{j}\right)= \begin{cases}1, & \text { if } C_{j}=C_{v r u} \\ 0, & \text { otherwise }\end{cases}
$$

3) Relative positioning: Let us note $\mathbf{L}_{r}$ the true position of an obstacle w.r.t. the vehicle.

The perception system calculates the relative location of the pedestrian (w.r.t. the vehicle), $\mathbf{L}_{r}^{\text {per }}(x, y)$, with a covariance $Q_{r}^{p e r}$ by the laser based detection system. The likelihood for positioning with perception follows the Gaussian distribution model $p\left(\mathbf{L}_{r}^{\text {per }} \mid \mathbf{L}_{r}, \boldsymbol{Q}_{r}^{\text {per }}\right)$.

The global positioning system, e.g. GPS, provides the absolute vehicle (resp. VRU) location, $\mathbf{L}_{a}\left(x_{v e h}, y_{v e h}\right)$ (resp. $\left.\mathbf{L}_{a}\left(x_{v r u}, y_{v r u}\right)\right)$, with a covariance $\boldsymbol{Q}_{a, v e h}\left(\right.$ resp. $\left.\boldsymbol{Q}_{a, v r u}\right)$. As a result of the V2P communication, the relative location of the pedestrian can be calculated, $\mathbf{L}_{r}^{\text {com }}\left(x_{v r u}-x_{v e h}, y_{v r u}-y_{v e h}\right)$ with covariance $\boldsymbol{Q}_{r}^{c o m}=\boldsymbol{Q}_{a, v e h}+\boldsymbol{Q}_{a, v r u}$, and the likelihood for positioning with communication follows the Gaussian distribution model $p\left(\mathbf{L}_{r}^{c o m} \mid \mathbf{L}_{r}, \boldsymbol{Q}_{r}^{\text {com }}\right)$. 


\section{MHT applied for VRU protection: preliminaries}

A road obstacle is represented by its relative position $\mathbf{L}_{r}[k]$, at a time step $k$, and its class, $C \in \mathcal{C} . \mathcal{C}=\left\{C_{j}, j=1, \ldots, N_{c}\right\}$ is a set of $N_{c}$ classes of road obstacles (e.g., pedestrian, vehicle,...).

Let us define a track as a temporal series of consecutive observations of a dynamic road obstacle in eq. (6).

$$
\mathbf{T}_{k}=\left(\mathbf{L}_{r}[n], C\right)_{n=1, \ldots, k}
$$

The perception system provides a set of obstacles detected around the vehicle, $\mathcal{T}_{k}^{\text {per }}=\left\{\mathbf{T}_{k, i}^{\text {per }}, i=1, \ldots, N_{k}^{\text {per }}\right\}$. The communication system provides a set of communicating obstacles, $\mathcal{T}_{k}^{c o m}=\left\{\mathbf{T}_{k, i}^{c o m}, i=1, \ldots, N_{k}^{c o m}\right\}$.

Inspired by multi-target tracking where association between imprecise observations and tracks is central, we propose to apply MHT [47] to tackle the issue of association between perception and V2P communication tracks. This method rely on a probabilistic framework to generate association hypotheses between the two types of obstacles.

Given a cumulative set of input tracks $\mathcal{T}_{k}=\left\{\mathbf{T}_{k, i}, i=\right.$ $\left.1, \ldots, N_{k}\right\}$, at time $\mathrm{k}$, the MHT generates a set of hypotheses $\Omega_{k}=\left\{\Omega_{k}^{j}, j=1, \ldots, J_{k}\right\}$ which associates the cumulative set of tracks with visible or hidden VRUs. As the set of input tracks is updated, $\Omega_{k}$ is generated by considering the possible combinations between input and output tracks.

An hypothesis $\Omega_{k}^{j}$ can be viewed as a joint hypothesis formed from a prior hypothesis $\Omega_{k-1}^{g}$ and an association hypothesis with the current data $\psi^{h}$. Hence, the probability of $\Omega_{k}^{j}$ given the measurements up to time $\mathrm{k}$ is given in eq. (7) using the recursive Bayesian formulation.

$$
\begin{aligned}
p\left(\Omega_{k}^{j} \mid \mathcal{T}_{k}\right)= & p\left(\Omega_{k-1}^{g}, \psi^{h} \mid \mathcal{T}_{k}\right) \\
= & \frac{1}{n} p\left(\mathcal{T}[k] \mid \Omega_{k-1}^{g}, \psi^{h}\right) \\
& \times p\left(\psi^{h} \mid \Omega_{k-1}^{g}\right) p\left(\Omega_{k-1}^{g} \mid \mathcal{T}_{k-1}\right)
\end{aligned}
$$

Here,

- $p\left(\mathcal{T}[k] \mid \Omega_{k-1}^{g}, \psi^{h}\right)$ is the likelihood of current tracks states $\mathcal{T}[k]$ given the association hypothesis,

- $p\left(\psi^{h} \mid \Omega_{k-1}^{g}\right)$ is the probability of current data association hypothesis given the prior hypothesis,

- $p\left(\Omega_{k-1}^{g} \mid \mathcal{T}_{k-1}\right)$ is the probability of the prior hypothesis given the past tracks states,

- $n$ is a normalization term which ensures $p\left(\Omega_{k}^{j} \mid \mathcal{T}_{k}\right)$ sums to 1 .

Fig. 5 illustrates the hypotheses generated from the two scenarios introduced by Fig 3. In LOS conditions (Fig. 5a), hypotheses $\Omega_{k}^{1}$ to $\Omega_{k}^{3}$ describe the associations of the communicating pedestrian with the three detected obstacles and hypothesis $\Omega_{k}^{4}$ represents the possibility that the communicating pedestrian is not detected. With $\Omega_{k}^{4}, \mathbf{T}_{k, 1}^{n p}$ is called the "not perceived" track. In NLOS conditions, hypotheses $\Omega_{k}^{1}$ to $\Omega_{k}^{3}$ describe the associations of the communicating pedestrian with the three detected obstacles and hypothesis $\Omega_{k}^{4}$ represents the possibility that the communicating pedestrian is not detected.

In this work, we apply MHT for the fusion of perception and V2P communication. More specifically, this paper focuses on establishing the likelihood term $p\left(\mathcal{T}[k] \mid \Omega_{k-1}^{g}, \psi^{h}\right)$ when the

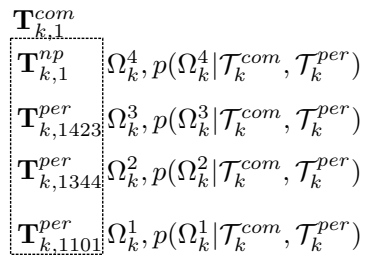

(a) LOS condition.

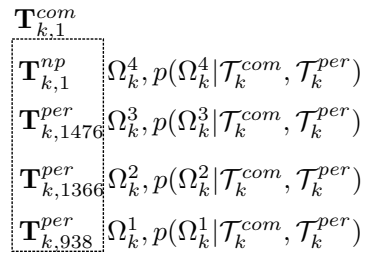

(b) NLOS condition.
Fig. 5: MHT example.

current tracks states $\mathcal{T}[k]$ are given by perception and $\mathrm{V} 2 \mathrm{P}$ communication systems. The reader interested in expressing the likelihood term $p\left(\psi^{h} \mid \Omega_{k-1}^{g}\right)$ should refer to [47].

\section{MHT applied for fusing perception and V2P communica-} tion

1) Definition: The a posteriori distribution $p\left(\Omega_{k}^{j} \mid \mathcal{T}_{k}^{\text {com }}, \mathcal{T}_{k}^{\text {per }}\right)$ of an hypothesis $\Omega_{k}^{j}$ is calculated by applying eq. (7) to the perception and communication tracks. In this section, the measurement likelihood term $p\left(\mathcal{T}_{k}^{\text {com }}, \mathcal{T}_{k}^{\text {per }} \mid \Omega_{k-1}^{g}, \psi^{h}\right)$ is expressed as a function of positioning, classification and possible occlusions.

2) Likelihood of perception to a communication tracks: Given a combination $\psi^{h}, N_{k}^{a s s, h}$ perception tracks are associated with communication tracks and $N_{k}^{n p, h}$ communicating obstacles are not perceived.

Let us define $\mathbf{T}_{k, i}^{a s s}=\left(\mathbf{T}_{k, l}^{p e r}, \mathbf{T}_{k, m}^{c o m}\right)$, a track generated by associating the perception track $\mathbf{T}_{k, l}^{p e r}$ with the communication track $\mathbf{T}_{k, m}^{c o m}$, and $\mathbf{T}_{k, i}^{n p}$ a track, called a "not perceived" track, created by a communication track, $\mathbf{T}_{k, m}^{c o m}$, and no perception track when the communicating VRU is located in NLOS condition w.r.t. the vehicle.

Without much loss of generality, we can assume that communicating VRUs are independent. Hence, the likelihood of perception and communication tracks, $p\left(\mathcal{T}_{k}^{\text {com }}, \mathcal{T}_{k}^{\text {per }} \mid \Omega_{k-1}^{g}, \psi^{h}\right)$, is expressed as the product of likelihoods for every association track, $\mathbf{T}_{k, i}^{a s s}$, between perception and communication, and likelihoods for every "not perceived" track, $\mathbf{T}_{k, i}^{n p}$ in eq. (8).

$$
\begin{aligned}
p\left(\mathcal{T}_{k}^{c o m}, \mathcal{T}_{k}^{\text {per }} \mid \Omega_{k-1}^{g}, \psi^{h}\right)= & \prod_{i=1}^{N_{k}^{a s s, h}} p\left(\mathbf{T}_{k, i}^{a s s} \mid \Omega_{k-1}^{g}, \psi^{h}\right) \\
& \times \prod_{i=1}^{N_{k}^{n p, h}} p\left(\mathbf{T}_{k, i}^{n p} \mid \Omega_{k-1}^{g}, \psi^{h}\right)
\end{aligned}
$$

The first term of eq. (8) considers the association of a perception track $\mathbf{T}_{k, l}^{p e r}$ with a communication track $\mathbf{T}_{k, m}^{c o m}$. The likelihood of such an association is ruled by the positioning of the communication obstacle w.r.t. the perception obstacle and the classification established by the perception system. This promotes the association of a communicating pedestrian with an obstacle which has a high chance of being a pedestrian. Hence, eq. (9) expresses the likelihood term $p\left(\mathbf{T}_{k, i}^{a s s} \mid \Omega_{k-1}^{g}, \psi^{h}\right)$ as the probability of the relative positioning of the communicating obstacle, $\mathbf{L}_{r, m}^{c o m}[k]$, given the relative positioning of the perception obstacle, $\mathbf{L}_{r, l}^{p e r}[k]$, and the covariance of positioning 
with communication, $\boldsymbol{Q}_{r, m}^{\text {com }}$, weighted by the probability that the class of the perception obstacle is the class, $C_{m}^{c o m}$, of the communication obstacle.

$$
\begin{aligned}
p\left(\mathbf{T}_{k, i}^{a s s} \mid \Omega_{k-1}^{g}, \psi^{h}\right)= & p\left(\mathbf{L}_{r, m}^{c o m}[k] \mid \mathbf{L}_{r, l}^{\text {per }}[k], \boldsymbol{Q}_{r, m}^{c o m}\right) \\
& \times P_{c}^{\text {per }}\left(C_{m}^{\text {com }}\right)
\end{aligned}
$$

The second term of eq. (8) considers the case of communicating obstacles which are "not perceived". The likelihood of such hypothesis depends on the ratio of the area within the statistical gate that is occluded as illustrated in Fig. 6.
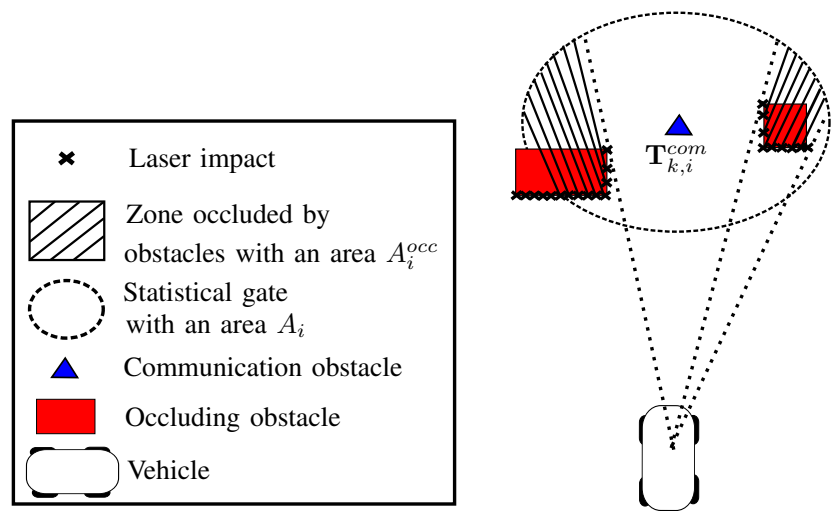

Fig. 6: Likelihood of "not perceived" track.

Let us define $A_{i}$ the area of the statistical gate, i.e., the zone where the VRU might be present, for the communication track $\mathbf{T}_{k, i}^{c o m}$ and $A_{i}^{o c c}$ the area of the occluded zone inside this gate. $A_{i}^{\text {occ }}$ can be calculated using the sensors data. Indeed, the point cloud produced by a laser scanner describes the limit of the vehicle FOV meaning that, for every laser point, the space between the point and the vehicle is visible as the space behind the point is occluded. Finally, eq. (10) formulates the likelihood term $p\left(\mathbf{T}_{k, i}^{n p} \mid \Omega_{k-1}^{g}, \psi^{h}\right)$ as the ratio between the area of occlusion, $A_{i}^{\text {occ }}$, and the total area, $A_{i}$, inside the gate, called ratio of occluded area.

$$
p\left(\mathbf{T}_{k, i}^{n p} \mid \Omega_{k-1}^{g}, \psi^{h}\right)=\frac{A_{i}^{o c c}}{A_{i}}
$$

3) A posteriori distribution formula: The a posteriori distribution $p\left(\mathbf{T}_{k, i}^{a s s} \mid \Omega_{k-1}^{g}, \psi^{h}\right)$ is calculated using eq. (8) for every hypothesis $\Omega_{k}^{j}$.

As the number of hypothesis is growing with the appearance of new perception and communication tracks, it is necessary to delete hypotheses with a probability below a given fixed threshold $\gamma_{f u s}$ in a so called pruning method.

\section{E. Estimation of VRUs state variables}

At each time instant $k$, an estimation of the state variables is taken from the hypothesis $\hat{\Omega}_{k}$ which has the highest $a$ posteriori distribution.

$$
\hat{\Omega}_{k}=\underset{j=1, \ldots, J_{k}}{\arg \max }\left(p\left(\Omega_{k}^{j} \mid \mathcal{T}_{k}^{\text {com }}, \mathcal{T}_{k}^{\text {per }}\right)\right)
$$

The estimation of the state of VRUs is done differently whether a track $\mathbf{T}_{k, l}^{p e r}$ is associated with a track $\mathbf{T}_{k, m}^{c o m}$ or a track $\mathbf{T}_{k, i}^{c o m}$ is "not perceived".
Concerning associated obstacles, dynamic parameters, in our case the relative position $\mathbf{L}_{r}$, are estimated from perception, and static parameters, in our case the obstacle class $C$, are estimated from V2P communication. Therefore, an associated track $\mathbf{T}_{k, i}^{a s s} \in \mathcal{T}_{k}^{a s s}$ is described in eq. (12) using parameters from $\mathbf{T}_{k, l}^{p e r}$ and $\mathbf{T}_{k, m}^{c o m}$.

$$
\mathbf{T}_{k, i}^{a s s}=\left(\mathbf{L}_{r, l}^{p e r}[n], C_{m}^{c o m}\right)_{n=1, \ldots, k}
$$

Concerning "not perceived" obstacles, only the parameters of the V2P communication VRU can be accessed. Consequently, a "not perceived" track $\mathbf{T}_{k, i}^{n p} \in \mathcal{T}_{k}^{n p}$ is described in eq. (13) using parameters of the corresponding V2P communication tracks, $\mathbf{T}_{k, i}^{c o m}$.

$$
\mathbf{T}_{k, i}^{n p}=\left(\mathbf{L}_{r, i}^{p e r}[n], C_{i}^{c o m}\right)_{n=1, \ldots, k}
$$

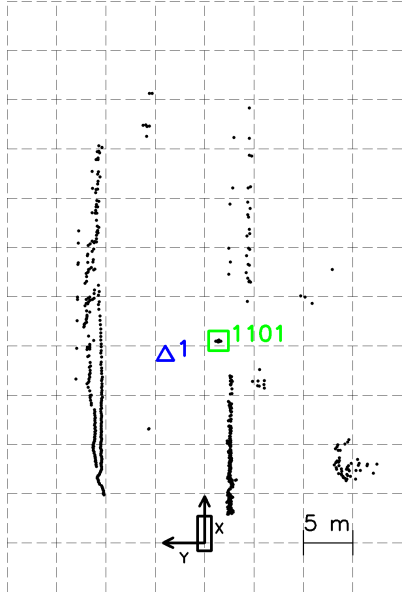

(a) LOS condition.

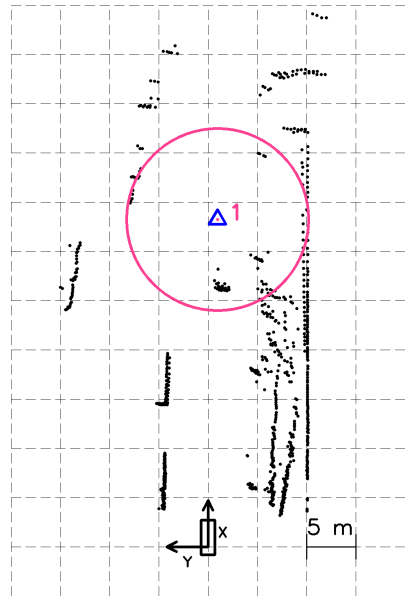

(b) NLOS condition.
$\Delta$ Communicating pedestrian

$$
\text { Associated track } O \text { "Not perceived" track }
$$

Fig. 7: Fusion between perception and V2P communication.

Fig. 7 illustrates the output of our fusion algorithm.

In LOS condition (Fig. 7a), an obstacle is classified as pedestrian by the perception system which generates an hypothesis with a high probability for associating the communicating pedestrian together with this obstacle. Therefore, the fusion step outputs an associated track (green square) in this situation.

In NLOS condition (Fig. 7b), no obstacle is classified as pedestrian by the perception system and an obstacle classified as vehicle generates many laser impacts inside the statistical gate. Consequently, the fusion step outputs a "not perceived" track (magenta circle) meaning that the communicating pedestrian cannot be seen in the sensor data.

\section{Evaluation}

\section{A. Experiment set-up}

We evaluate our system in real conditions on the Inria ParisRocquencourt campus as illustrated in Fig. 8. 


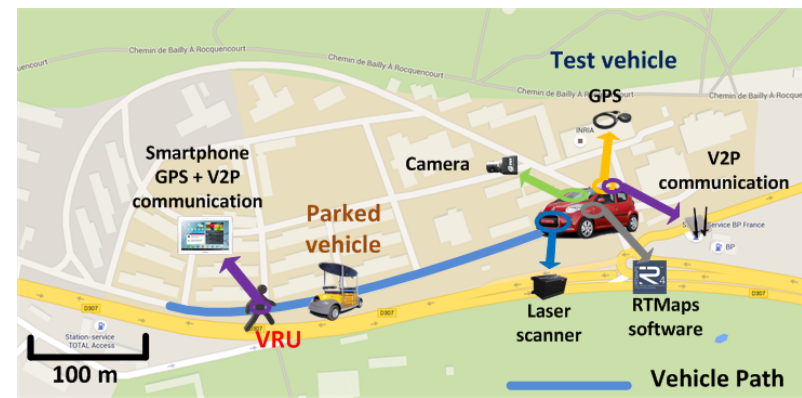

Fig. 8: Experiment configuration

In the experiments, a vehicle approaches a pedestrian from more than $150 \mathrm{~m}$ of distance with the speed of appx. $4 \mathrm{~m} / \mathrm{s}$ in the following two cases:

- no obstacle exists between the vehicle and the pedestrian (LOS),

- pedestrian stands behind a parked vehicle, with height of $2 \mathrm{~m}$ (NLOS).

Table III lists the functionalities, which are implemented in the vehicle and the pedestrian's hand-held device.

TABLE III: Experiment configuration

\begin{tabular}{|c|c|}
\hline Vehicle & $\begin{array}{l}\text { Laser based obstacle detection [6]: } \\
\text { - Angular resolution: } 0.25^{\circ} \\
\text { - Frequency: } 10 \mathrm{~Hz} \\
\text { - Range: } 100 \mathrm{~m}\end{array}$ \\
\hline Pedestrian & V2ProVu application for pedestrian protection [23] \\
\hline $\begin{array}{l}\text { Common } \\
\text { Features }\end{array}$ & $\begin{array}{l}\text { Wi-Fi communication: } \\
\text { - Ad-hoc mode } \\
\text { - Standard IEEE } 802.11 \mathrm{~g} \\
\text { - Transmission power: } 24 \mathrm{dBm} \\
\text { - Data rate: } 1 \mathrm{Mbps} \\
\text { GPS-based positioning: } \\
\text { - Frequency: } 1 \mathrm{~Hz}\end{array}$ \\
\hline
\end{tabular}

On the vehicle side, the local position of the vehicle is estimated based on new laser measurement measurements [48] and fused with the current GPS position to broadcast CAM packets every $100 \mathrm{~ms}$. On the pedestrian side, the procedures described in Fig. 2 are made.

LOS results are made of six experiments as NLOS results are composed of five experiments. Hence, our system has been tested in total in eleven scenarios containing the two possible conditions.

For performance investigation, the distance between the vehicle and the pedestrian is split into distant bins and the average performances are evaluated for the individual bins.

Detection probability for both perception and communication and positioning accuracy with V2P communication have been investigated in [8]. In this paper, we focus on evaluating perception and communication fusion.

\section{B. Theoretical aspects of perception/communication fusion}

The fusion error is calculated in every of the distance bins defined for the evaluation. The true state of the pedestrian is used as ground truth when it is available, otherwise, the distance error is calculated by a penalty term obtained from positioning error established in [8].
Fig. 9 shows the fusion error for the perception system, the communication system, and the theoretical perception/communication fusion system for all experiments. As stated in [8], communication system performs better for long distances and perception system is preferred for low distances.

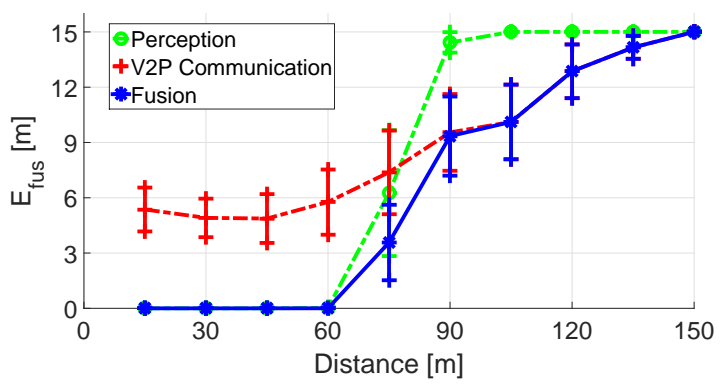

Fig. 9: Fusion error with individual systems

A perfect perception/communication fusion system should benefit from both advantages to have an error equivalent to the error with communication at large distances and decrease this error at low distances.

Since the experimental scenarios are not all the same, the detection and positioning may vary from one test to another leading to a high confidence interval at medium range, i.e. between 60 and $90 \mathrm{~m}$. The cooperative system is particularly interesting at this range because a correct combination of perception and communication should reduce this confidence interval and have a lower error than the standalone systems.

\section{Detecting LOS and NLOS conditions}

In these experiments, a safety system has to detect a pedestrian who is either in LOS or NLOS conditions w.r.t. the vehicle. The cooperative VRU protection system has the capability to deal with both situations by calculating the likelihood of an association between a perception and a communication track (eq. (9)) and the likelihood for a communication track of being "not perceived" (eq. (10)). Hence, when a single pedestrian is present, the system can be seen as a detection system between LOS and NLOS conditions. To evaluate the impact of each likelihood function, we investigate the obstacle classification performances which influences eq. (9) and the occlusion area ratio within the statistical gate which rules eq. (10).

1) Influence of obstacle classification: The perception system classifies pedestrians and non pedestrians based on geometric observations (width, length,...) and the communication system relies on the received V2P data to classify these users.

A correct classification of the true pedestrian as a pedestrian is called a true positive (TP), as a classification of another obstacle as pedestrian is called a false positive (FP). True Positive Rate (TPR) is determined by the number of correct classifications over the number of detection of the pedestrian, and, False Positive Rate (FPR) is defined as the number of false positives over the total number of non pedestrian obstacles.

Receiving Operator Characteristics (ROC) is a major metric that displays TPR vs FPR and evaluates detection systems. 


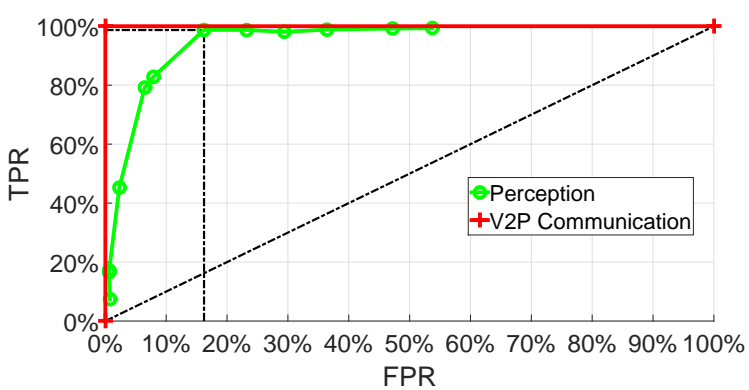

Fig. 10: Receiving operator characteristics

Fig. 10 shows the ROC curves for the perception system only and for V2P communication system only.

By varying the parameters, e.g., the expected width, length, number of impacts of pedestrians in laser sensor data, the number of TP and FP are modified with the perception system and the ROC curve is calculated for the perception. The perception system can almost perfectly classify the pedestrian at the cost of a high FPR, TPR is greater than $93 \%$ for FPR greater than $30 \%$.

As VRUs are assumed to inform correctly their class with the application installed in their hand-held device, the ROC curve with the V2P communication system is ideal with $100 \%$ of TPR at $0 \%$ of FPR.

Having too many FP may lead to incorrect associations with MHT as some obstacles can be considered as the communicating pedestrian. Having a lower number of TP may lead to take the decision that a pedestrian is "not visible" when it is detected by the perception system but not classified correctly. Finally, as the fusion relies on a classification score in eq. (9), good performances of the perception system is required for the fusion algorithm. Any classification error will degrade the ideal fusion error introduced in this paper. It is worth noting that our perception system is quite simple and computationally not expensive. If we want to get better performances, using vision techniques can be a complementary solution [5].

2) Influence of occlusion: The ratio of occluded area appears in eq. (10) as the parameter which controls the weight of the hypothesis for a "not perceived" pedestrian. With our system, this ratio can be calculated from laser measurements by counting the ratio of laser impacts which are in front of the statistical gate over the maximum number of laser impacts which could fall in this gate.

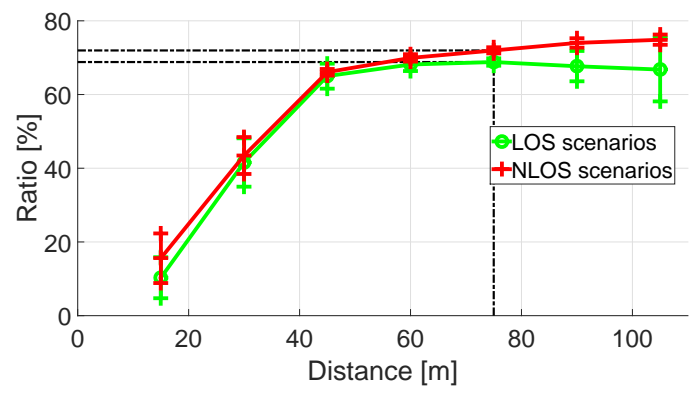

Fig. 11: Ratio of Occluded Area
Fig. 11 shows the ratio of occluded area versus the vehiclepedestrian distance for LOS and NLOS scenarios. In every condition, this value decreases as the vehicle-pedestrian distance reduces.

NLOS conditions do not have much influence on the occluded area ratio. Only $2 \%$ of difference is seen between LOS and NLOS conditions at $75 \mathrm{~m}$. This is because, our parked vehicle has a width of $1.5 \mathrm{~m}$ which is small compared to the gate of the communication track which has a diameter of $15 \mathrm{~m}$.

In summary, in both LOS and NLOS conditions,

- when the distance is high, likelihood that the obstacle is occluded is high, resulting in more chance to conclude that the communicating obstacle is "not perceived",

- when the distance is low, likelihood that the obstacle is occluded is low, resulting in more chance to associate the communicating obstacle with a perception track.

\section{Applicability of the cooperative protection system}

In Fig. 9, we showed the theoretical application error with a perception/communication fusion. In real conditions, this application error can be greater than the theoretical one due to an association between the communicating pedestrian and an incorrect perception obstacle. Fig. 12 illustrates the experimental application error for both LOS and NLOS scenarios.

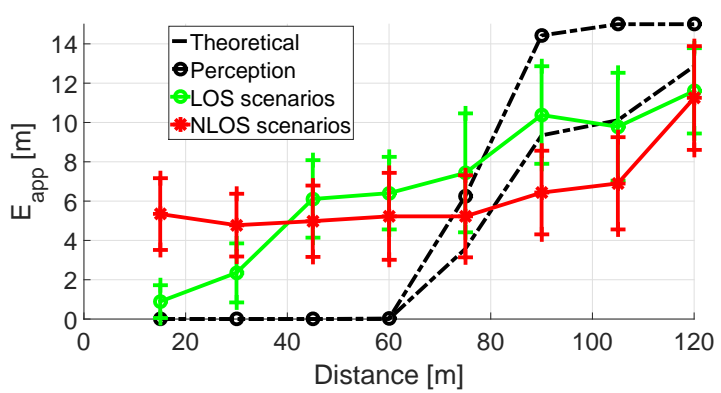

Fig. 12: Fusion error with the cooperative system

In LOS scenarios, experimental results show the application error decreases down to $0.9 \mathrm{~m}$ with vehicle-pedestrian distance. However, the existence of a large amount of obstacle within the gate of the communicating pedestrian can lead to incorrect associations during the fusion step. Particularly, at distances above $40 \mathrm{~m}$, the pedestrian is not always recognized. Therefore, the communicating pedestrian may be associated to resembling obstacles such as trees or posts as shown by the higher value of the fusion error between 40 and $60 \mathrm{~m}$. Despite the imprecision of the cooperative system, it enhances the fusion error of the perception system only for distances above $70 \mathrm{~m}$ and it has the ability to detect pedestrians who are not visible from the vehicle.

In NLOS scenarios, different weather conditions (less clouds and no rain) during the experiments have conducted to better performances at long range. In these cases, the occlusion by the parked vehicle generates associations with wrong obstacles, especially below $40 \mathrm{~m}$, when the influence of occlusion is low (see Fig. 11). Finally, the error is of $5 \mathrm{~m}$ at $30 \mathrm{~m}$ range. Improving the accuracy of the positioning system 
and the modeling of the occlusion term would allow to reduce the fusion error in NLOS conditions.

\section{E. Discussion}

In this paper, we introduced an approach for fusing perception V2P communication data by weighting information from these two systems and selecting the one with highest probability. A more selective approach could have been to choose the input information source based on the distance as it was shown in Fig. 9 that V2P communication distance performs better for distances above $70 \mathrm{~m}$ and perception system is better below this distance. However, such approach would be efficient only when the VRU is visible from the vehicle as the proposed system can also handle the case where the VRU is hidden by an obstacle.

In addition, the cooperative VRU protection system provide redundant information to benefit from each individual system in various conditions:

- compared to a system based only on perception, the cooperative protection system is able to give an alert even when a pedestrian is not visible w.r.t. the vehicle,

- compared to a system based only on communication, the cooperative approach is able to overcome the limitations of GPS localization system by having an error of less than $3 \mathrm{~m}$ below $30 \mathrm{~m}$ of distance.

However, our approach still lacks of precision for correctly identifying a communicating pedestrian due to imprecision of the classification with perception and due to an imprecise GPS localization with V2P communication.

Here, only the communicating pedestrian is assumed to be present in the surrounding environment of the vehicle. However, in real conditions, "not communicating" VRU can also be present and should be detected by a protection system. Thus, more research is needed to distinguish different kind of users and make the correct associations between perception and communication data. Adding new features for describing VRUs, using sensors such as cameras both on the vehicles and in the road infrastructure could be of interest to improve the performances of such cooperative approaches.

\section{CONCLUSION}

This paper introduces a new cooperative system for VRU protection that exploits the strengths of both the perception and communication mechanisms. We present a method for information fusion between perception and V2P communication. This study shows that an ideal fusion between perception and V2P communication should benefit from both systems. We showed accurate classification by the perception and precise absolute positioning with the communication systems are the main limitations of current approaches.

Our future work includes 1) an extension of our system to more complex scenarios, i.e., with more VRUs and vehicles 2) development of fusion mechanisms which can deal with information that may not be temporally synchronized due to communication delays 3 ) investigation of the LDM for a more efficient management of the information provided by the internal vehicle system and by vehicular communications and 4) benchmark of the proposed approach with commercial pedestrian detection system.

\section{ACKNOWLEDGMENT}

The authors are grateful to Evangeline POLLARD for her precious advice. This work benefits from a financial support of French funding "financement par le plan d'Avenir".

\section{REFERENCES}

[1] E. Commission, "ITS \& Vulnerable Road Users," http: //ec.europa.eu/transport/themes/its/road/action_plan/its_and_vulnerable_ road users en.htm, 2012, [Online; access le 2013-04-19].

[2] WHO, "Global Status Report On Road Safety 2013: supporting a decade of action," World Health Organization, Tech. Rep., 2013.

[3] T. Gandhi and M. Trivedi, "Pedestrian Protection Systems: Issues, Survey, and Challenges," IEEE Transactions on Intelligent Transportation Systems, vol. 8, no. 3, pp. 413-430, Sep. 2007.

[4] D. Gerónimo, A. M. López, A. D. Sappa, and T. Graf, "Survey of pedestrian detection for advanced driver assistance systems." IEEE transactions on pattern analysis and machine intelligence, vol. 32, no. 7, pp. 1239-58, 2010.

[5] P. Dollar, C. Wojek, B. Schiele, and P. Perona, "Pedestrian Detection: An Evaluation of the State of the Art," IEEE Transactions on Pattern Analysis and Machine Intelligence, vol. 34, no. 4, pp. 743-761, 2012.

[6] G. Gate, A. Breheret, and F. Nashashibi, "Centralized fusion for fast people detection in dense environment," in IEEE International Conference on Robotics and Automation (ICRA), 2009.

[7] P. Merdrignac, E. Pollard, and F. Nashashibi, "2D Laser Based Road Obstacle Classification For Road Safety Improvement," in IEEE International Workshop on Advanced Robotics and its Social Impacts (ARSO), 2015.

[8] P. Merdrignac, O. Shagdar, I. Ben Jemaa, and F. Nashashibi, "Study on Perception and Communication Systems for Safety of Vulnerable Road Users," in 18th IEEE International Conference on Intelligent Transportation Systems (ITSC), 2015.

[9] M. Tons and R. Doerfler, "Radar sensors and sensor platform used for pedestrian protection in the EC-funded project SAVE-U," in IEEE Intelligent Vehicles Symposium (IV), 2004.

[10] K. Manston, "The Challenges Of Using Radar For Pedestrian Detection," in The 16th JCT Traffic Signal Symposium, 2011.

[11] B. Clarke, S. Worrall, G. Brooker, and E. Nebot, "Towards mapping of dynamic environments with FMCW radar," in IEEE Intelligent Vehicles Symposium (IV), 2013.

[12] F. Bu and C. Y. Chan, "Pedestrian Detection in Transit Bus Application: Sensing Technologies and Safety Solutions," in IEEE Intelligent Vehicles Symposium (IV), 2005.

[13] A. Ewald and V. Willhoeft, "Laser scanners for obstacle detection in automotive applications," in IEEE Intelligent Vehicles Symposium (IV), 2000.

[14] K. C. Fuerstenberg, K. C. J. Dietmayer, and V. Willhoeft, "Pedestrian recognition in urban traffic using a vehicle based multilayer laserscanner," in Proceedings of the IEEE Intelligent Vehicles Symposium, 2002, 2002.

[15] B. Schwarz, "LIDAR: Mapping the world in 3-D," Nature Photonics, vol. 4, no. 7, 2010

[16] D. M. Gavrila, J. Giebel, and S. Munder, "Vision-based pedestrian detection: the PROTECTOR system," in IEEE Intelligent Vehicles Symposium (IV), 2004.

[17] I. P. Alonso, D. F. Llorca, M. A. Sotelo, L. M. Bergasa, P. Revenga De Toro, J. Nuevo, M. Ocana, and M. A. Garcia Garrido, "Combination of feature extraction methods for SVM pedestrian detection," IEEE Transactions on Intelligent Transportation Systems, vol. 8, no. 2, pp. 292-307, 2007.

[18] M. Enzweiler and D. M. Gavrila, "Monocular Pedestrian Detection: Survey and Experiments," IEEE Transactions on Pattern Analysis and Machine Intelligence, vol. 31, no. 12, pp. 2179-2195, 2009.

[19] M. Bertozzi, A. Broggi, and P. Grisleri, "Pedestrian detection in infrared images," in IEEE Intelligent Vehicles Symposium (IV), 2003.

[20] C. Bellotti, F. Bellotti, A. De Gloria, L. Andreone, and M. Mariani, "Developing a near infrared based night vision system," in IEEE Intelligent Vehicles Symposium (IV), 2004.

[21] K. David and A. Flach, "Car-2-X and pedestrian safety," IEEE Vehicular Technology Magazine, vol. 5, no. 1, pp. 70-76, 2010. 
[22] ETSI TS 102 637-2; Intelligent Transport Systems (ITS); Vehicular communications; Basic set of Applications; Part 2: Specification of Cooperative Awareness Basic Service, Std., Mar. 2011, v1.2.1.

[23] J. Anaya, P. Merdrignac, O. Shagdar, F. Nashashibi, and J. Naranjo, "Vehicle to Pedestrian Communications for Protection of Vulnerable Road Users," in IEEE Intelligent Vehicle Symposium (IV), 2014.

[24] ETSI TS 102 637-3; Intelligent Transport Systems (ITS); Vehicular communications; Basic set of Applications; Part 3: Specifications of Decentralized Environmental Notification Basic Service, Std., Sept. 2010, v1.1.1.

[25] EU, Directive 2010/40/EU of the European Parliament and of the Council, Std., July 2010, 2008/0263/COD.

[26] ISO 21217:2010; Intelligent transport systems - Communications access for land mobiles (CALM) - Architecture, Std., Apr. 2010.

[27] ETSI TR 102 638; Intelligent Transport Systems (ITS); Vehicular communications; Basic set of Applications, Std., Sept. 2010, v1.0.4.

[28] IEEE Standard for Information technology - Telecommunications and information exchange between systems - Local and metropolitan area networks - Specific requirement, Part 11: Wireless LAN Medium Access Control (MAC) and Physical Layer (PHY) Specifications, IEEE Computer Society Std., July 2010, iEEE Std 802.11p-2010.

[29] C. Sugimoto, Y. Nakamura, and T. Hashimoto, "Prototype of pedestrianto-vehicle communication system for the prevention of pedestrian accidents using both $3 \mathrm{G}$ wireless and WLAN communication," in $3 \mathrm{rd}$ International Symposium on Wireless Pervasive Computing, 2008.

[30] D. Ikeda, M. Horie, R. Yamaguchi, T. Wada, and H. Okada, "An Effective Detection Algorithm of the Relative Movement between Vehicles and Pedestrians in VPEC," in Second ACM international workshop on Wireless network testbeds, experimental evaluation and characterization, 2007.

[31] M. Bagheri, M. Siekkinen, and J. K. Nurminen, "Cellular-based Vehicle to Pedestrian ( V2P ) Adaptive Communication for Collision Avoidance," in 3rd International Conference on Connected Vehicles \& Expo (ICCVE), 2014

[32] ETSI TS 102 863; Intelligent Transport Systems (ITS); Vehicular communications; Basic set of Applications; Local Dynamic Map (LDM); Rationale for and guidance on standardization, Std., June 2011, v1.1.1.

[33] ETSI TS 102 636-1; Intelligent Transport Systems (ITS); Vehicular communications; GeoNetworking; Part 1: Requirements, Std., Mar 2010, v1.1.1.

[34] H. Li, M. Tsukada, F. Nashashibi, and M. Parent, "Multivehicle Cooperative Local Mapping: A Methodology Based on Occupancy Grid Map Merging," IEEE Transactions on Intelligent Transportation Systems, pp. $1-12,2014$.

[35] G. Challita, S. Mousset, F. Nashashibi, and A. Bensrhair, "An application of $\mathrm{V} 2 \mathrm{~V}$ communications : Cooperation of vehicles for a better car tracking using GPS and vision systems," in IEEE Vehicular Networking Conference (VNC), 2009

[36] E. Pollard and D. Gingras, "Improved Low Cost GPS Localization By Using Communicative Vehicles," in 12th International Conference on Control, Automation, Robotics and Vision (ICARCV), 2012.

[37] M. Obst, L. Hobert, and P. Reisdorf, "Multi-Sensor Data Fusion for Checking Plausibility of V2V Communications by Vision-based Multiple-Object Tracking," in IEEE Vehicular Networking Conference $(V N C), 2014$

[38] N. E. Zoghby, V. Cherfaoui, and T. Denoeux, "Evidential Distributed Dynamic Map for Cooperative Perception in VANets," in IEEE Intelligent Vehicles Symposium (IV), 2014.

[39] S. Demmel, "Building an Augmented Map for Road Risk Assessment Sébastien Demmel," Ph.D. dissertation, Centre for Accident Research and Road Safety, Queensland University of Technology, 2013.

[40] E. D. Kaplan and C. J. Hegarty, Understanding GPS: Principles and Applications. Artech house, London UK, 2006.

[41] M. Liebner, F. Klanner, and C. Stiller, "Active Safety for Vulnerable Road Users based on Smartphone Position Data," in IEEE Intelligent Vehicles Symposium (IV), 2013.

[42] A. Sikora, "Communication and Localization for a Cooperative eSafetySystem," in 4th IEEE Workshop on Intelligent Data Acquisition and Advanced Computing Systems: Technology and Applications, 2007.

[43] D. Lill, M. Schappacher, A. Gutjahr, and A. Sikora, "Development of a wireless communication and localization system for VRU eSafety," in 7th International Symposium on Communication Systems Networks and Digital Signal Processing (CSNDSP), 2010.

[44] B. Fardi, U. Neubert, N. Giesecke, H. Lietz, and G. Wanielik, "A fusion concept of video and communication data for VRU recognition," in 11th International Conference on Information Fusion, 2008.
[45] H. Kloeden, D. Schwarz, E. M. Biebl, and R. H. Rasshofer, "Effectiveness study of cooperative sensor systems for VRU-safety," in IEEE Intelligent Vehicles Symposium (IV), 2012.

[46] Y. Bar-Shalom and X.-R. Li, Multitarget-multisensor tracking: principles and techniques. Storrs, CT: University of Connecticut, 1995, vol. 19.

[47] D. B. Reid, "An algorithm for tracking multiple targets," IEEE Transactions on Automatic Control, vol. 24, no. 6, 1979.

[48] Z. Alsayed, G. Bresson, F. Nashashibi, and A. Verroust-Blondet, "Pmlslam: a solution for localization in large-scale urban environments," in PPNIV-IROS 2015, 2015

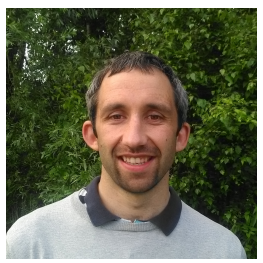

Pierre Merdrignac received his master of engineering from Telecom Bretagne, Brest, in 2012 and his Ph.D. in robotics from Mines ParisTech in 2015 From 2012 to 2015, he was a Ph.D. student in RITS Team at INRIA Paris and in VEDECOM institute. Since 2015 , he is a research engineer at INRIA. His research interest includes environment perception, multi-source information fusion, and vehicular communication. He is a member of IEEE since 2016.

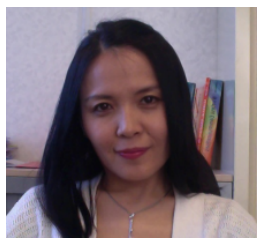

Oyunchimeg Shagdar Oyunchimeg Shadar received her B.S., M.S., and Ph.D. degrees in Electric and Electronic System Engineering, from Nagoya University of Technology, in 2002, 2004 and 2009, respectively. From 2004 to 2012, she has been has been been working at the ATR Adaptive Communications Research Laboratories. She is a researcher of RITS team at INRIA Paris since 2012 and a researcher in VEDECOM institute since 2015. Her research interest includes wireless communications, especially medium access control, routing/forwarding/access point selection, and transmission coding for vehicular, mesh, sensor applications. She is a member of IEEE since 2008.

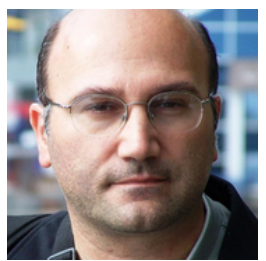

Fawzi Nashashibi 48 years, is a senior researcher and the Program Manager of RITS Team at INRIA Paris since 2010. He has been senior researcher and Program Manager in the robotics center of the Ecole des Mines de Paris (Mines ParisTech) since 1994 and was an $\mathrm{R} \& \mathrm{D}$ engineer and a project manager at ARMINES since May 2000. He was previously a research engineer at PROMIP (working on mobile robotics perception dedicated to space exploration) and a technical manager at Light Co. where he led the developments of Virtual Reality/Augmented Reality applications. Fawzi Nashashibi has a Masters Degree in Automation, Industrial Engineering and Signal Processing (LAAS/CNRS), a $\mathrm{PhD}$ in Robotics from Toulouse University prepared in (LAAS/CNRS) laboratory, and a HDR Diploma (Accreditation to research supervision) from University of Pierre et Marie Curie (Paris 6). His main research topics are in environment perception and multi-sensor fusion, vehicle positioning and environment 3D modeling with main applications in Intelligent Transport Systems and Robotics. He played key roles in more than 50 European and national French projects such as Carsense, HAVE-it, PICAV, CityMobil, ARCOS, ABV, LOVe, SPEEDCAM,... some of which he has coordinated. He is also involved in many collaborations with French and international academics and industrial partners. He is author of numerous publications and patents in the field of ITS and ADAS systems. His current interest focuses on advanced urban mobility through the design and development of highly Automated Transportation Systems. This includes Highly Automated Unmanned Guided Vehicles (such as Cybercars) as well automated personal vehicles. In this field he is known as an international expert. Since 1994 he is also a lecturer in several universities (Mines ParisTech, Paris 8 Saint-Denis, Leonard de Vinci Univ. - ESILV professor, Telecom Sud Paris, INT Evry, Ecole Centrale dElectronique) in the fields of image and signal processing, 3D perception, 3D infographics, mobile robotics and C++/JAVA programming. He is also member of the ITS Society and the Robotics \& Automation Society. He is an Associate Editor of several IEEE international conferences such as ICRA, IROS, IV, ITSC, ICARCV 